\title{
Research progress on the antibacterial mechanisms of carvacrol: a mini review
}

\author{
Dan Zhang ${ }^{1}$, Ren-You Gan ${ }^{*}$, Ying-Ying Ge1, Qiong-Qiong Yang ${ }^{1}$, Jiao Ge1, Hua-Bin \\ $\mathbf{L i}^{2}$, Harold Corke ${ }^{1^{*}}$
}

${ }^{1}$ Department of Food Science \& Technology, School of Agriculture and Biology, Shanghai Jiao Tong University, Shanghai 200240, China; ${ }^{2}$ Guangdong Provincial Key Laboratory of Food, Nutrition, and Health, Department of Nutrition, School of Public Health, Sun Yat-Sen University, Guangzhou 510080, China

Corresponding author: Dr. Ren-You Gan, Department of Food Science \& Technology, School of Agriculture and Biology, Shanghai Jiao Tong University, Shanghai 200240, China

Submission Date: August 23 ${ }^{\text {rd }}$, 2018, Acceptance Date: October $28^{\text {th }}$, 2018, Publication Date: October $30^{\text {th }}, 2018$

Citation: Zhang D., Gan R., Ge Y., Yang Q., Ge J., Li H., Corke H. Research Progress on the Antibacterial Mechanisms of Carvacrol: A Mini Review. Bioactive Compounds in Health and Disease 2018; 1(6): 71-81. DOI: https://doi.org/10.31989/bchd.v1i6.551

\begin{abstract}
Background: Carvacrol is an aromatic phenolic terpenoid widely existing in the volatile oils of thyme, oregano, and some other aromatic plants. Recent studies have found that carvacrol possesses excellent antibacterial activity.

Thus, in order to provide an updated information about the antibacterial potentials of carvacrol we summarized recent publications about the antibacterial activity of carvacrol, with special attention paid to its antibacterial molecular mechanisms, including disrupting cell membrane, depleting intracellular ATP, inducing reactive oxygen species, inhibiting efflux pumps, as well as suppressing two important virulence factors: biofilm and quorum sensing. In conclusion, carvacrol is a promising natural antibacterial compound with potential application in food preservation and infection.
\end{abstract}

Keywords: Carvacrol, antibacterial mechanisms, biofilm, quorum sensing 

INTRODUCTION

Antibiotics are one of the greatest discoveries of the 20th century and have been used to treat infectious diseases and promote the growth of animals as food additives for a long time [1, 2]. However, the abuse of antibiotics has led to severe problems, such as the emergence of drug-resistant bacteria, antibiotic residues, and environmental pollution. Therefore, increasing attention has been paid to seeking for desirable antibiotic alternatives.

Plant essential oils are attractive antibiotic substitutes, since they are natural, have no residue, and difficult to induce drug resistance [3]. Among them, oregano (Origanum vulgare) essential oil has been widely used as food preservatives and feed additives for antiseptic and antibacterial effects in Europe and the United States due to its quite high antibacterial activity [4]. Carvacrol (Figure 1), also known as 5-isopropyl-2-methylphenol, is an oxygenated monoterpenoid. Carvacrol is the major bioactive compound in oregano essential oil, accounting for $70 \%-80 \%$. Carvacrol has a broad antibacterial spectrum against Escherichia (E.) coli [5], Staphylococcus (S.) aureus [6], Listeria (L.) monocytogenes [7], and Salmonella (S.) typhimurium [8]. Significantly, carvacrol has been reported not to influence or even positively increase the growth of beneficial intestinal bacteria, including Lactobacillus, Bifidobacteria, and Firmicutes, at the concentration of Clostridium inhibition, providing theoretical evidence for its use in feed additives and clinical applications [9-12]. To provide a better understanding of the antibacterial potential of carvacrol, we summarize and discuss its antibacterial mechanisms.

Figure 1. The chemical structure of carvacrol.

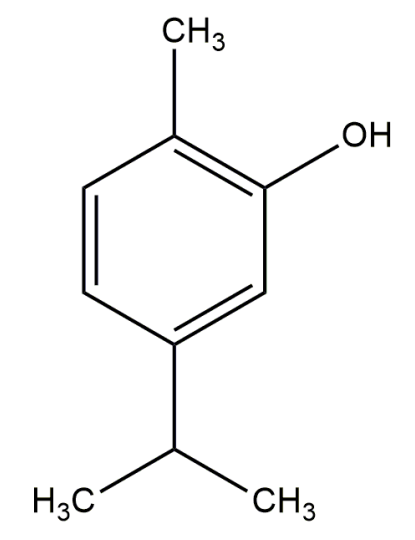

\section{ANTIBACTERIAL MECHANISMS}

\section{The disruption of cell membranes}

Bacterial cell membranes form barriers against the permeation of antibiotics and chemical fungicides and avoid the phagocytosis of the body's immune system [13]. Studies have suggested that the disruption of cytoplasmic membrane is an important mechanism for the antibacterial activity of carvacrol (Figure 2), seriously influencing the fluidity, integrity, and functionality of 

cell membranes [14, 15].

Wang et al. found that the content of membrane unbranched fatty acids (UBFAs) in Gram-positive S. aureus ATCC 43300 rose from $34.9 \%$ to $62.37 \%$, compensating for the increased fluidizing effects caused by carvacrol at $0.77 \mathrm{mM}$. More serious membrane morphological damage, such as destruction of integrity could be observed in the presence of higher concentrations (from 1.03 to $4.12 \mathrm{mM}$ ) of carvacrol [16]. Additionally, another study also revealed that exposure of $S$. aureus to the sub-lethal concentration of carvacrol $(0.6 \mu \mathrm{L} / \mathrm{mL})$ resulted in the increased absorbance at $260 \mathrm{~nm}$, indicating that membrane damage caused the leakage of intracellular substances, and the subsequent dysregulation of membrane-related protein secretion system further accelerated the bacterial death [17]. Additionally, it was reported that compounds with the octanol/water partition coefficient $(\log \mathrm{P})$ value higher than 3 had intrinsic hydrophobic properties, showing high affinity to cell membranes and causing physical changes, such as the order and stability of membrane phospholipid bilayer [18] and the $\log \mathrm{P}$ value of carvacrol is 3.62 [19]. The hydrophobic nature of carvacrol should play an important role in the loss of bacterial membrane integrity and influence the proteins embedded, like proteins that form efflux pumps, disrupting the normal physiological function of related organelle [20]. Moreover, carvacrol has been suggested to have a stronger antibacterial effect on gram-positive bacteria than gram-negative bacteria, probably due to the later rich in hydrophilic lipopolysaccharide in the cell membrane [21]. Therefore, the findings above support that carvacrol can target bacterial cell membranes and destroy membrane-related functions.

Figure 2. The schematic diagram of carvacrol destroying bacterial cell membrane

- Carvacrol

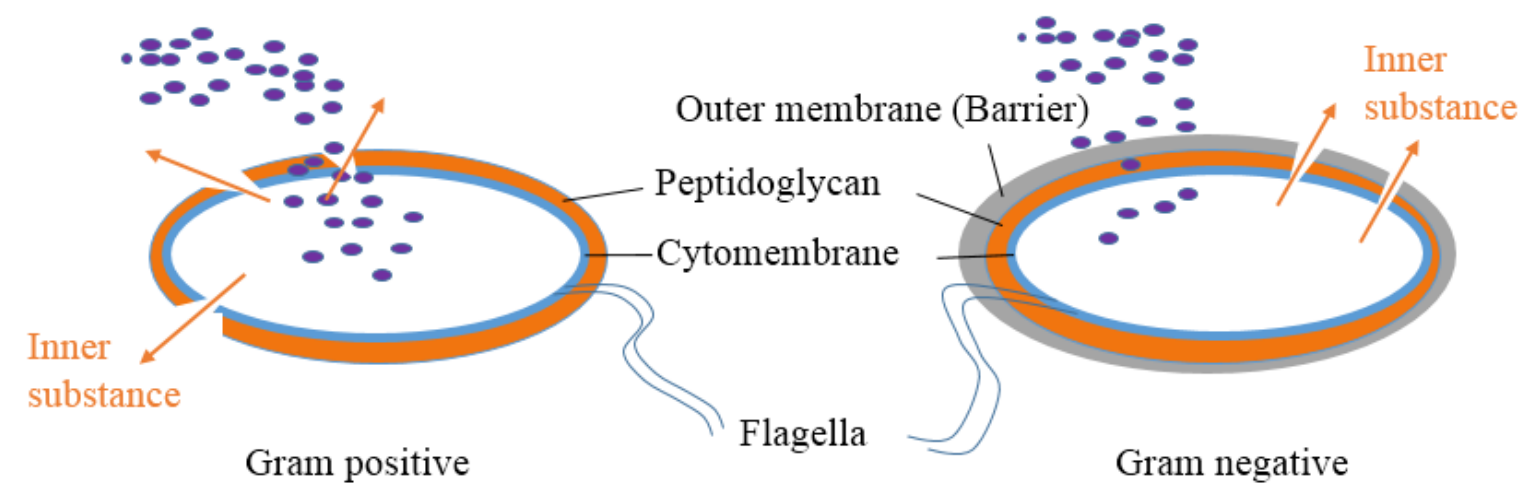

\section{Depletion of intracellular ATP}

The existing free hydroxyl and delocalized electronic systems of carvacrol have also been proven to be essential for its antibacterial activity (Figure 3) [18]. When carvacrol enters the cytoplasm of bacteria, the delocalized lone electron pair on the hydroxyl oxygen of carvacrol can form a $p-\pi$ 

conjugate with its benzene ring, promoting the release of proton on the phenolic hydroxyl group into the bacterial cytoplasm, thereby increasing the acidity of bacterial cytoplasm. However, the normal cytoplasmic $\mathrm{pH}$ is generally close to neutral. In order to maintain the appropriate $\mathrm{pH}$ inside the cell, bacteria have to transport excessive proton out of the cell accompanied with the consumption of ATP, finally depleting the intraceullular ATP pool and leading to the cell death [22]. Therefore, depletion of intracellular ATP is an important antibacterial mechanism of carvacrol.

\section{Induction of Reactive oxygen species (ROS)}

Previous studies found that limonene and some bactericidal antibiotics shared similar antibacterial mechanisms by inducing ROS, such as hydroxyl radicals [23, 24]. In addition to fluorine in nature, hydroxyl radicals demonstrate high oxidizing power, which can easily destroy proteins, lipid membranes, and DNA, leading to cell death [25].

Carvacrol has been reported to induce ROS production in bacteria (Figure 3). At a concentration of $450 \mu \mathrm{g} / \mathrm{mL}$, carvacrol was reported to have elevated ROS levels in E. coli by fluorescence imaging [26]. Additionally, Chueca et al. found that $100 \mu \mathrm{L} / \mathrm{L}$ of carvacrol reduced the amount of $E$. coli by over $2.5 \operatorname{logs}$ in $3 \mathrm{hr}$ under aerobic conditions, but the reduction could be controlled within $1 \mathrm{log}$ by adding a ROS scavenger thiourea. Compared with the control, the $\triangle r e c A$ mutant of $E$. coli was found to be more sensitive to carvacrol, indicating that carvacrol caused oxidative damage to DNA because RecA could induce SOS responses in normal strains with DNA damage [27]. In the presence of carvacrol at $15 \mu \mathrm{g} / \mathrm{mL}$, increased oxidative stress was also found in Streptococcus mutans, verified by the downregulated expression of superoxide dismutase gene [28]. Overall, ROS plays an important role in carvacrol-induced cell damage, even cell death.

\section{Inhibition of efflux pump}

The efflux pump is a chromosome-encoded translocator that is located on the plasma membrane of bacteria. Many bacteria can extrude the antibacterial drugs to the extracellular environment through the efflux pump system, thereby reducing the intracellular drug concentration and causing the antibiotic resistance of bacteria [29]. Plant products are natural sources of the efflux pump inhibitor (EPI). Recent studies found that carvacrol can be a potential EPI of bacteria (Figure 3).

Miladi et al. demonstrated that carvacrol inhibited the efflux of ethidium bromide (EB) in the food-borne pathogens $S$. aureus ATCC 25923 and E. coli ATCC 35218 in a dose-dependent manner; the combination of carvacrol and tetracycline at sub-inhibitory concentrations also increased the sensitivity of the test bacteria to antibiotics [30]. Additionally, by using the DNA microarray-based transcriptomics assay, the transcriptional levels of multidrug efflux pump 

regulatory genes, such as acrA and $m d t M$, were significantly altered in E. coli MG1655 treated with $100 \mathrm{mg} / \mathrm{L}$ of carvacrol [31]. The results above indicate that carvacrol can be used as an EPI to increase the sensitivity of the test strain to antibacterial agents and made the bacteria more easily cleared.

Figure 3. The schematic diagram of carvacrol depleting intracellular ATP (a), inducing reactive oxygen species (b), and inhibiting efflux pump (c).

- Carvacrol

- Reactive oxygen species (ROS)

$\Delta$ Hydrogen ion a

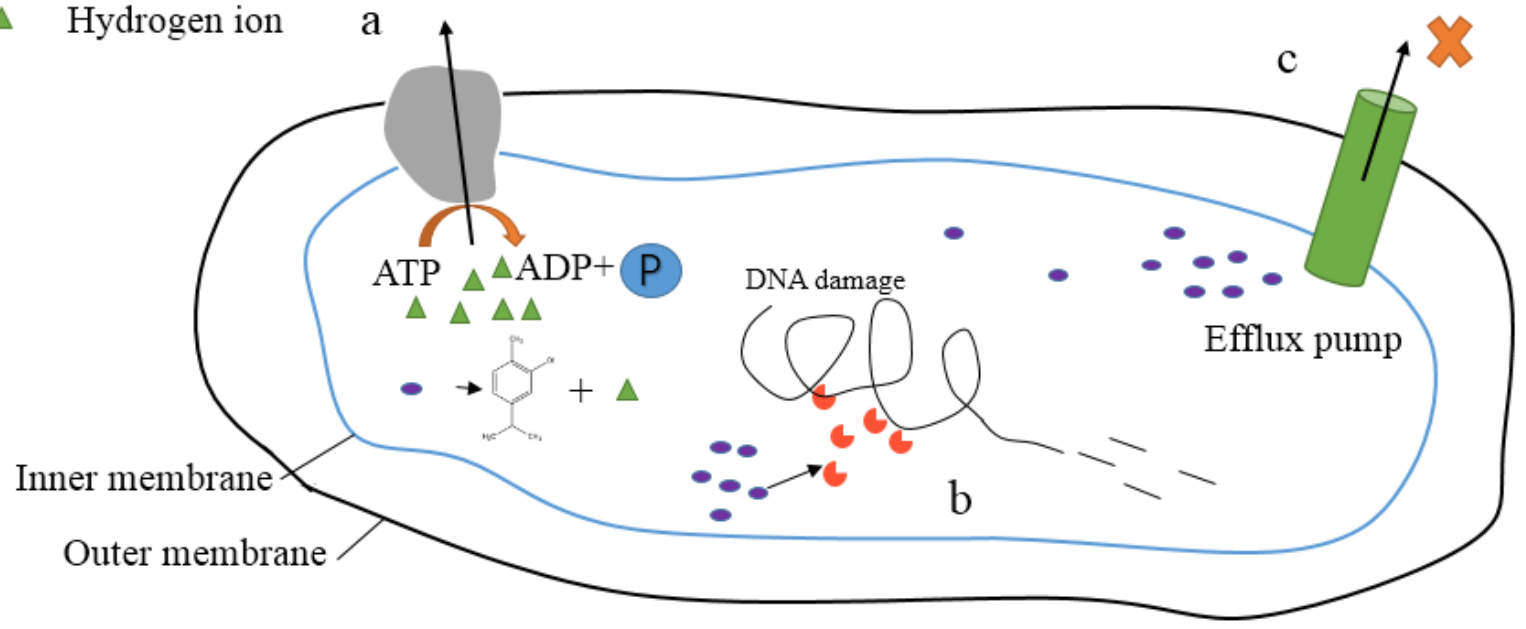

\section{Inhibition of bacterial biofilm}

Many bacteria have the ability to adhere to biotic or abiotic surfaces and then proliferate, differentiate, and secrete a series of polysaccharide-protein complexes, which envelop the bacterial community to form a membrane of organized aggregates of bacteria, called biofilm. The biofilm is highly resistant to antibiotics and the host immune defense systems [23].

More recently, the antibiofilm activity of carvacrol has been reported in many bacteria. These bacteria include Gram-positive bacteria like S. aureus [32], Streptococcus mutans [28], and L. monocytogenes [33], in addition to Gram-negative bacteria like S. typhimurium [34], Pseudomonas (P.) aeruginosa [35], and E. coli [36]. The diagram of this mechanism is displayed in Figure 4. The inhibitory effect of carvacrol on the growth of the biofilm is related to its intrinsic characteristics. The solubility of carvacrol in water is $800 \pm 10 \mathrm{~mL} / \mathrm{L}$ and the relative hydrophilicity allows carvacrol to diffuse through the polar polysaccharide matrix of the biofilm. Additionally, the general hydrophobicity promotes carvacrol to specifically interact with cell membranes, affecting their structures and functions [22, 37].

Significantly, carvacrol can not only inhibit the initial biofilm formation, but also eradicate pre-formed biofilms. Higher concentrations are required to remove the pre-formed biofilm since 

the viscous matrix and complex three-dimensional structures of biofilm inhibit the permeation and diffusion of carvacrol in the pre-formed biofilms. The existence of dormant cells embedded in biofilm possess higher resistant ability [38, 39]. For example, the biofilm formed by $S$. typhimurium was reduced by the presence of carvacrol at sub-lethal concentrations $(<0.5 \mathrm{mM})$. However, there was little influence on pre-formed biofilm, even up to $8 \mathrm{mM}$ [40]. Additionally, many studies have linked the antibiofilm ability of carvacrol to some following factors. (1) Carvacrol decreased bacterial motility and adhesion to the surface [41]. For example, the disruption of flagellin synthesis was found in E. coli O157:H7 treated with a sub-lethal concentration of carvacrol, coupled with decreased biofilm formation [42]. (2) Carvacrol reduced bacterial density caused by delayed cell growth in planktonic cells and subsequently reduced the quorum sensing signaling [32]. (3) Carvacrol directly killed bacteria to induce the biofilm destruction [43]. Overall, carvacrol exhibits anti-biofilm activity with diverse rationales.

Figure 4. Schematic diagram of carvacrol suppressing biofilm (a) and quorum sensing. (b).

- Carvacrol

$\checkmark$ Signal molecules
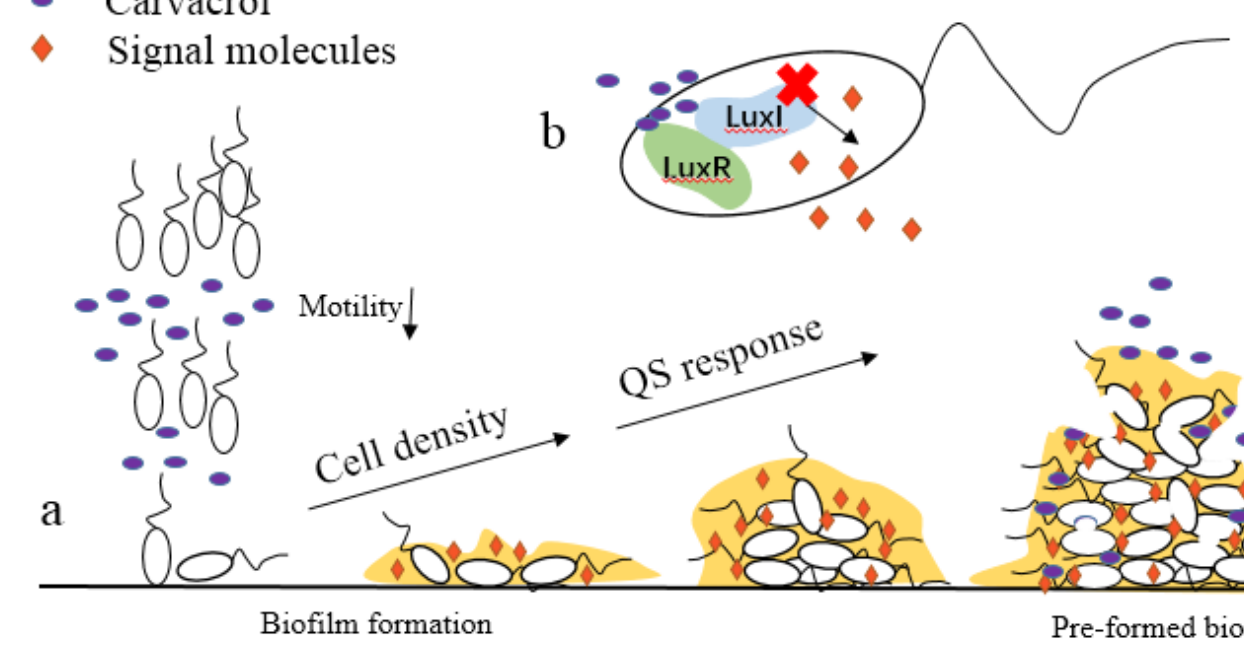

Biofilm formation

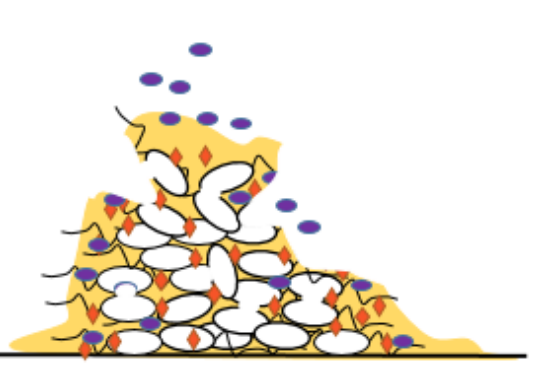

Pre-formed biofilm

\section{Blocking of quorum sensing}

Quorum sensing (QS) is a regulatory system of bacterial biological behavior based on population density and has received increasing attention in recent years [44]. Bacteria can synthesize and secrete numerous small molecules, which are also known as auto-inducers (AIs). With the accumulation of these signals, many bacterial behaviors can be activated, such as bioluminescence, toxin production, motility, and biofilm formation. Most Gram-negative bacteria use membrane-permeable fatty acyl homoserine lactone (AHLs) derivatives as signal molecules, which are synthesized by LuxI homologue and recognized by LuxR homologous, activating the transcription of downstream target genes [45].

In recent years, carvacrol has been highlighted as a desirable natural quorum sensing 

inhibitor (QSI) (Figure 4). In the work of Rodrigue et al., the QS-mediated phenotypes like pyocyanin production and biofilm formation in $P$. aeruginosa dose-dependently decreased by carvacrol [46]. Additionally, Janak et al. found that carvacrol formed $\pi-\pi$ bond and hydrogen bond with phenylalanines residues in ExpI (LuxI homolog) and lysine residue in the receptor protein ExpR (LuxR homolog) respectively, inhibiting the production of signaling molecules and activation of receptors in Pectobacterium species, with biofilm formation and other virulence factors mediated by QS also inhibited [47]. Moreover, Myszka et al. reported that carvacrol was able to inhibit the synthesis of AHLs, finally interfering with the QS signaling [48]. Overall, carvacrol is effective in QS inhibition and its natural and nontoxic characteristics make it suitable for further applications.

\section{CONCLUSIONS}

In conclusion, carvacrol has a broad spectrum of antibacterial activity, with the disruption of the cell membrane, induction of ROS, and inhibition of efflux pump, biofilm, and QS system as the main antibacterial mechanisms. Therefore, it is safe to use carvacrol as antimicrobial agents in the field of medical care or animal feeding. However, most of the previous studies focused on normal foodborne pathogens, while the antibacterial efficiency and related mechanisms of carvacrol on drug-resistant bacteria have not been as investigated. Additionally, most of the previous studies used the in vitro experiments to test the antibacterial effects of carvacrol.

Thus, its effects on animals and humans need further elucidation. Since the $\mathrm{LD}_{50}$ of carvacrol tested in vivo ranges from $310 \mathrm{mg} / \mathrm{kg}$ to $2700 \mathrm{mg} / \mathrm{kg}$, much higher than the doses used in the antibacterial filed [22], it is reasonable to speculate that carvacrol can be generally safe within its antibacterial concentration. Furthermore, the enhancement of antibacterial activity needs to be studied in the future. For example, through nanotechnology. Moreover, while the antibacterial mechanisms of carvacrol have been initially proposed, its molecular targets and regulated signaling pathways still need further identification. Overall, carvacrol exhibits promising antibacterial activity and can be good candidates as food preservatives and antibacterial agents with medical applications.

Acknowledgments and Funding: The study was financially supported by the the National Key R\&D Program of China (2017YFC1600100), Shanghai Pujiang Talent Plan (No. 18PJ1404600), and Shanghai Basic and Key Program (No. 18JC1410800).

Author's Contributions: D.Z. and R.Y.G. developed the concept and drafted the manuscript. Y.Y.G., Q.Q.Y., J.G., H.B.L., and H.C. edited and revised the manuscript. The final version was approved by all authors. 
Conflicts of Interest: All authors declare no conflict of interest.

\section{REFERENCES}

1. Allen HK, Levine UY, Looft T, Bandrick M, Casey TA: Treatment, promotion, commotion: antibiotic alternatives in food-producing animals. Trends Microbiol 2013, 21(3):114-119.

2. Seal BS, Lillehoj HS, Donovan DM, Gay CG: Alternatives to antibiotics: a symposium on the challenges and solutions for animal production. Anim Health Res 2013, 14(1):78-87.

3. Bassanetti I, Carcelli M, Buschini A, Montalbano S, Leonardi G, Pelagatti P, Tosi G, et al.: Investigation of antibacterial activity of new classes of essential oils derivatives. Food Control 2016.

4. Lukas B, Schmiderer C, Novak J: Essential oil diversity of European Origanum vulgare L. (Lamiaceae). Phytochem 2015, 119:32-40.

5. Stratakos AC, Sima F, Ward P, Linton M, Kelly C, Pinkerton L, Stef L, et al.: The in vitro, effect of carvacrol, a food additive, on the pathogenicity of $\mathrm{O} 157$ and non-O157 Shiga-toxin producing Escherichia coli. Food Control 2017, 84.

6. Engel JB, Heckler C, Tondo EC, Daroit DJ, Malheiros PDS: Antimicrobial activity of free and liposome-encapsulated thymol and carvacrol against Salmonella, and Staphylococcus aureus, adhered to stainless steel. Int J Food Microbio 2017, 252:18-23.

7. Chung D, Cho TJ, Rhee MS: Citrus fruit extracts with carvacrol and thymol eliminated 7-log acid-adapted Escherichia coli O157:H7, Salmonella typhimurium, and Listeria monocytogenes: A potential of effective natural antibacterial agents. Food Res Int 2018, 107:578.

8. Carvalho RI, Medeiros ASDJ, Chaves M, Souza ELD, Magnani M: Lipids, pH, and their interaction affect the inhibitory effects of carvacrol against Salmonella typhimurium PT4 and Escherichia coli O157:H7. Front Microbiol 2017, 8.

9. Liu XZ, Diarra MS, Zhang YG, Wang Q, Yu H, Nie SP, Xie MY, et al.: Effect of encapsulated carvacrol on the incidence of necrotic enteritis in broiler chickens. Avian Pathol 2016, 45(3):357.

10. Mooyottu S, Flock G, Upadhyay A, Upadhyaya I, Maas K, Venkitanarayanan K: Protective Effect of Carvacrol against Gut Dysbiosis and Clostridium difficile Associated Disease in a Mouse Model. Front Microbiol 2017, 8.

11. Mooyottu S, Kollanoorjohny A, Flock G, Bouillaut L, Upadhyay A, Sonenshein AL, Venkitanarayanan K: Carvacrol and trans-Cinnamaldehyde Reduce Clostridium difficile Toxin Production and Cytotoxicity in Vitro. Int J Mol Sci 2014, 15(3):4415-4430.

12. Yin DF, Du EC, Yuan JM, Gao JX, Wang YL, Aggrey SE, Guo YM: Supplemental thymol and carvacrol increases ileum Lactobacillus population and reduces effect of 

necrotic enteritis caused by Clostridium perfringes in chickens. Sci Rep 2017, 7(1):7334.

13. Bo-Ping LI, Wen-Chun HU: Review on the bioactivity of carvacrol. Journal of Longdong University 2017.

14. Hammer KA, Heel KA: Use of multiparameter flow cytometry to determine the effects of monoterpenoids and phenylpropanoids on membrane polarity and permeability in staphylococci and enterococci. Int J Antimicrob Ag 2012, 40(3):239.

15. Vasconcelos SECB, Melo HM, Cavalcante TTA, Catunda FEA, de Carvalho MG, Menezes FGR, de Sousa OV, et al.: Plectranthus amboinicus, essential oil and carvacrol bioactive against planktonic and biofilm of oxacillin- and vancomycin-resistant Staphylococcus aureus. Bmc Complem Altern M 2017, 17(1):462.

16. Wang LH, Wang MS, Zeng XA, Zhang ZH, Gong DM, Huang YB: Membrane destruction and DNA binding of Staphylococcus aureus cells induced by carvacrol and its combined effect with a pulsed electric field. J Agr Food Chem 2016, 64(32):6355-6363.

17. Souza EL, Oliveira CEV, Stamford TLM, Conceicao ML, Gomes Neto NJ: Influence of carvacrol and thymol on the physiological attributes, enterotoxin production and surface characteristics of Staphylococcus aureus strains isolated from foods. Braz J Microbiol 2013, 44(1):29-35.

18. Ben Arfa A, Combes S, Preziosi-Belloy L, Gontard N, Chalier P: Antimicrobial activity of carvacrol related to its chemical structure. Lett Appl Microbiol 2010, 43(2):149-154.

19. Nostro A, Roccaro AS, Bisignano G, Marino A, Cannatelli MA, Pizzimenti FC, Cilni, PL, et al.: Effects of oregano, carvacrol and thymol on Staphylococcus aureus and Staphylococcus epidermidis biofilms. J Med Microbiol 2007, 56(4):519.

20. Cirino ICS, Menezessilva SMP, Silva HTD, de Souza EL, Siqueira JP: The essential oil from Origanum vulgare L. and its individual constituents carvacrol and thymol enhance the effect of tetracycline against Staphylococcus aureus. Chemotherapy 2015, 60(5):290-293.

21. Shen SX, Zhang TH, Yuan Y, Lin SY, Xu JY, Ye HQ: Effects of cinnamaldehyde on Escherichia coli, and Staphylococcus aureus membrane. Food Control 2015, 47(47): 196-202.

22. Suntres ZE, Coccimiglio J, Alipour M: The bioactivity and toxicological actions of carvacrol. Crit Rev Food Sci 2015, 55(3):304.

23. Chueca B, Pagan R, Garcíagonzalo D: Differential mechanism of Escherichia coli inactivation by (+)-limonene as a function of cell physiological state and drug's concentration. Plos One 2014, 9(4):e94072.

24. Kohanski MA, Dwyer DJ, Hayete B, Lawrence CA, Collins JJ: A common mechanism of cellular death induced by bactericidal antibiotics. Cell 2007, 130(5):797.

25. Chen CS, Chao HT, Pan RL, Wei YH: Hydroxyl radical-induced decline in motility and 
increase in lipid peroxidation and DNA modification in human sperm. Iubmb Life 2010, 43(2):291-303.

26. Khan I, Bahuguna A, Kumar P, Bajpai VK, Kang SC: Antimicrobial potential of carvacrol against uropathogenic Escherichia coli via membrane disruption, depolarization, and reactive oxygen species generation. Front Microbiol 2017, 8.

27. Chueca B, Pagan R, Garciagonzalo D: Oxygenated monoterpenes citral and carvacrol cause oxidative damage in Escherichia coli without the involvement of tricarboxylic acid cycle and Fenton reaction. Int J Food Microbiol 2014, 189: 126-131.

28. Khan ST, Khan M, Ahmad J, Wahab R, Abd-Elkader OH, Musarrat J, Alkhathlan HZ, et al.: Thymol and carvacrol induce autolysis, stress, growth inhibition and reduce the biofilm formation by Streptococcus mutans. Amb Express 2017, 7(1):49.

29. Alav I, Sutton JM, Rahman KM: Role of bacterial efflux pumps in biofilm formation. J Antimicrob Chemoth 2018.

30. Miladi H, Zmantar T, Chaabouni Y, Fedhila K, Bakhrouf A, Mandouani K, Chaieb K: Antibacterial and efflux pump inhibitors of thymol and carvacrol against food-borne pathogens. Microb Pathog 2016, 99:95-100.

31. Chueca B, Perez-Saez E, Pagán R, Garcia-Gonzalo D: Global transcriptional response of Escherichia coli MG1655 cells exposed to the oxygenated monoterpenes citral and carvacrol. Int J Food Microbiol 2017, 257: 49.

32. Espina L, Pagan R, Lopez D, Garcia-Gonzalo D: Individual constituents from essential oils inhibit biofilm mass production by multi-drug resistant Staphylococcus aureus. Molecules 2015, 20(6):11357-72.

33. Desai MA, Soni KA, Nannapaneni R, Schilling MW, Silva JL: Reduction of Listeria monocytogenes biofilms on stainless steel and polystyrene surfaces by essential oils. J Food Prot 2012, 75(7):1332-1337.

34. Miladi H, Zmantar T, Kouidhi B, Chaabouni Y, Mandouani K, Bakhrouf A, Chaieb K: Use of carvacrol, thymol, and eugenol for biofilm eradication and resistance modifying susceptibility of Salmonella enterica serovar Typhimurium strains to nalidixic acid. Microb Pathogenesis 2017, 104:56-63.

35. Soumya E, Saad IK, Hassan L, Ghizlane Z, Hind M, Adnane R: Carvacrol and thymol components inhibiting Pseudomonas aeruginosa adherence and biofilm formation. Afr $\mathrm{J}$ Microbiol Res 2011, 5(20):3229-3232.

36. Campana R, Baffone W: Carvacrol efficacy in reducing microbial biofilms on stainless steel and in limiting re-growth of injured cells. Food Control 2018, 90:10-17.

37. Rodrigues JBD, de Souza NT, Alcantara JOA, de Sousa JM, Lira MC, de Figueiredo RCBQ, de Souza EL, et al.: Efficacy of using oregano essential oil and carvacrol to remove young and mature Staphylococcus aureus, biofilms on food-contact surfaces of stainless steel. LWT 2018, 93. 
38. Jadhav S, Shah R, Bhave M, Palombo EA: Inhibitory activity of yarrow essential oil on Listeria planktonic cells and biofilms. Food Control 2013, 29(1):125-130.

39. Sandasi M, Leonard CM, Viljoen AM: The in vitro antibiofilm activity of selected culinary herbs and medicinal plants against Listeria monocytogenes. Lett Appl Microbiol 2010, 50(1):30-35.

40. Burt SA, Ojofakunle VTA, Woertman J,Veldhuizen EJA: The natural antimicrobial carvacrol inhibits quorum sensing in Chromobacterium violaceum and reduces bacterial biofilm formation at sub-lethal concentrations. Plos One 2014, 9(4):e93414.

41. Gutierrez-Pacheco MM, Gonzalez-Aguilar GA, Martinez-Tellez MA, Lizardi-Mendoza J, Madera-Santana TJ, Bernal-Mercado AT, Vazquez-Armenta FJ, et al.: Carvacrol inhibits biofilm formation and production of extracellular polymeric substances of Pectobacterium carotovorum, subsp. carotovorum. Food Control 2018, 89.

42. Burt SA, van der Zee R, Koets AP, de Graaff AM, van Knapen F, Gaastra W, Haagsman HP, et al.: Carvacrol induces heat shock protein 60 and inhibits synthesis of flagellin in Escherichia coli O157:H7. Appl Environ Microb 2007, 73(14):4484-90.

43. Vasconcelos SECB, Melo HM, Cavalcante TTA, Catunda FEA, de Carvalho MG, Menezes FGR, de Sousa OV, et al.: Plectranthus amboinicus essential oil and carvacrol bioactive against planktonic and biofilm of oxacillin- and vancomycin-resistant Staphylococcus aureus. Bmc Complem Altern M 2017, 17(1):462.

44. Gokalsin B, Aksoydan B, Erman B, Sesal NC: Reducing virulence and biofilm of Pseudomonas aeruginosa, by potential quorum sensing inhibitor carotenoid: zeaxanthin. Microbial Ecology 2017, 74(2):1-8.

45. Chang CY, Krishnan T, Wang H, Chen Y, Yin WF, Chong YM, Tan LY, et al.: Non-antibiotic quorum sensing inhibitors acting against $\mathrm{N}$-acyl homoserine lactone synthase as druggable target. Sci Rep 2014, 4:7245.

46. Tapia-Rodriguez MR, Hernandez-Mendoza A, Gonzalez-Aguilar GA, Martinez-Tellez MA, Martins CM, Ayala-Zavala JF: Carvacrol as potential quorum sensing, inhibitor of Pseudomonas aeruginosa, and biofilm production on stainless steel surfaces. Food Control 2017, 75:255-261.

47. Joshi JR, Khazanov N, Senderowitz H, Burdman S, Lipsky A, Yedidia I: Plant phenolic volatiles inhibit quorum sensing in pectobacteria and reduce their virulence by potential binding to ExpI and ExpR proteins. Sci Rep 2016, 6.

48. Myszka K, Schmidt MT, Majcher M, Juzwa W, Olkowica M, Czaczyk K: Inhibition of quorum sensing-related biofilm of Pseudomonas fluorescens, KM121 by Thymus vulgare essential oil and its major bioactive compounds. Int Biodeter Biodegr 2016, 114:252-259. 